\title{
Performative Kompetenz und Fremdsprachenunterricht
}

\author{
Wolfgang Hallet
}

\begin{abstract}
Zusammenfassung
Der vorliegende Beitrag geht von der Theatralität und Performativität allen kulturellen Handelns aus und betrachtet den Fremdsprachenunterricht als einen Sonderfall von inszenierter Wirklichkeit. Lebensweltliche Situationen sind von Selbst-Repräsentationen und Inszenierungen geprägt, die in erheblichem Maße zur Identitätsfindung und -entwicklung im Sinne der Positionierung des Selbst im sozialen Raum und zur Konstitution sozialer Interaktion beitragen. Insofern gleichen lebensweltliche (Selbst-)Inszenierungen den literarischen Inszenierungen sozialer Handlungen in dramatischen Texten. Diese können im Hinblick auf die Entwicklung einer performativen lebensweltlichen Kompetenz als positive oder negative Modelle personaler und sozialer Inszenierungen und als Modelle interpersonaler Konfigurationen gesehen werden. Der Fremdsprachenunterricht wird als ein Ort verstanden, der zur Ausbildung der performativen Kompetenz der Lernenden beitragen kann.
\end{abstract}

\section{Die Performativität und Theatralität kulturellen Handelns}

'Performative Kompetenz' ist kein Begriff, der bisher bildungstheoretisch begründet worden oder Bestandteil eines eingeführten Kompetenzmodells ist, weder in fremdsprachlichen noch in anderen schulpädagogischen Kontexten. ${ }^{1}$ Ziel dieses Beitrags ist daher eine Darstellung des Konzepts mit einer umfassenderen Definition im Schlussteil. An dieser Stelle soll 'Performative Kompetenz' vorläufig definiert werden als ein Bündel von Fähigkeiten des Individuums, die Inszeniertheit allen sozialen Handelns zu verstehen, selbst soziale Interaktionssituationen zu initiieren, diese selbstbestimmt mitzugestalten und die eigene Rolle darin kritisch zu reflektieren. Die bildungstheoretische Definition einer solchen Kompetenz rekurriert darauf, dass Alltagshandlungen prinzipiell als theatral und performativ gelten können. Denn diskursiv-kommunikative Äußerungen sind nicht nur propositionale Aussagen, sondern, wie es die

\footnotetext{
${ }^{1}$ Der vorliegende Beitrag beruht auf den Überlegungen zur Performativen Kompetenz in: Hallet 2007, Hallet \& Hebel 2007 und vor allem auf der ausführlichen Darstellung des Konzepts in Hallet 2008. Eine knappe Zusammenfassung findet sich bei Hallet 2010a.
} 
Sprechakttheorie begründet hat, immer zugleich 'Akte' oder 'Handlungen', die soziokulturelle Wirklichkeit konstituieren. Sozio-kulturelle Wirklichkeit 'existiert' demnach nicht einfach, sondern sie wird von den in ihr auftretenden Aktanten in einem komplexen, interaktiven Zusammenspiel unablässig herbeigeführt, inszeniert, aufs Neue konstituiert, also erst in der Interaktion geschaffen. Anders ausgedrückt: Auch Alltagshandlungen weisen starke Züge der Inszenierung, der bewussten oder unbewussten In-Szene-Setzung, also des Theatralen und des Performativen auf, indem auf dem Wege der diskursiven und der nicht-diskursiven Interaktion soziale Situationen und die Anteile der jeweiligen Teilnehmer/innen daran jeweils aufs Neue konstituiert werden. Ein kleines Beispiel für eine Familiensituation, wie sie in einem Lehrwerkdialog (Klasse 7) modelliert ist (Green Line 3: 51), soll das verdeutlichen. Zunächst der kurze Dialog (Ausschnitt, alle Hervorhebungen im Original):

"(In the Patterson's living room. Mel and Amy are playing cards. Jake is listening to music on his MP3 player and playing air guitar to himself. Their parents enter the room.)

Mum: We're leaving now to see Grandpa. Will you be OK by yourselves? (Mel and Amy go on playing cards.) I said ... (shouts) WILL YOU BE OK BY YOURSELVES?Jake: You needn't shout, Mum. I'm 16, remember?Dad: We're taking our mobiles, so if there's a problem, just call.Mum: You'll look after the girls, won't you, Jake? Jake: Sure. I'll make sure they brush their teeth and go to bed early.Mel: Shut up, Jake. We're not babies?Dad: Bye everyone, and please be nice to each other! (Mr and Mrs Patterson leave.)"

Ohne dass dieser Dialog hier im Detail analysiert werden kann, ist doch auf Anhieb erkennbar, dass diese Situation, wie im Alltag auch, durch den 'Auftritt' der Eltern deutlich als 'Szene' mit einem 'Anfang', einem konfliktartigen Verlauf und einem Ende (dem 'Abgang' der Eltern) markiert ist. Eine bestehende Situation wird durch den Auftritt neuer Akteure, durch eine Unterbrechung von gerade stattfindenden Handlungen und vor allem durch die Eröffnung eines neuen Diskurses umdefiniert, wodurch eine neue Situation konstituiert wird. Hier ist auch sehr gut sichtbar, dass die Akteure einerseits sehr stark auf ihre (generationellen und geschlechterspezifischen) 'Rollen' und Verantwortlichkeiten festgelegt sind als Eltern, Kinder, Geschwister, Mann und Frau, Mädchen und Junge; andererseits werden diese Rollen und die ihnen zugrundeliegenden soziokulturellen und ethischen Setzungen in dieser Szene mindestens unterschwellig befragt und verhandelt; denn die Kinder weisen die Vorstellung von den 'aufsichtsbedürftigen', nicht selbstverantwortlichen 'Erziehungsbedürftigen' und die damit verbundenen Rollenzuweisungen durch die Eltern entweder ironisch oder explizit zurück, sodass die Szene auch den Charakter einer sozialen Aushandlung und eines damit verbundenen sozialen Konfliktes annimmt.

Wenngleich es sich um einen Lehrwerkdialog handelt, dessen Realismus diskutiert werden müsste, so verdeutlicht dieser kleine Szene doch sehr gut, 
dass sich familiäre Rollen, Eltern-Kind-Beziehungen und

Geschlechteridentitäten in genau dieser Weise in der Alltagsinteraktion und in den mit ihr verbundenen sozialen und sprachlich-diskursiven Aushandlungen herausbilden. Was Judith Butler für die re-iterative Herausbildung der Geschlechteridentität beschreibt (vgl. Butler 2002), lässt sich daher verallgemeinern: Identitäten, Selbstkonzepte und - komplementär dazu Wahrnehmungen des Gegenübers, also des ego und des alter, bilden sich auf dem Wege ständiger Re-Inszenierung in lebensweltlicher Alltagsinteraktion performativ heraus.

'Performativ' ist dies zu nennen in einem zweifachen Sinn: Zum einen werden Rollen und Identitäten in der Interaktion konstituiert ('performativ' also gemäß dem Performativitätsbegriff der Sprechakttheorie), und zum anderen weisen solche sozialen Interaktionen deutliche strukturelle Merkmale dramatischer 'performance' auf, wie man an der kleinen Szene oben sehen kann. Der Ethnologe Victor Turner hat es so beschrieben:

"Human beings belong to a species well endowed with means of communication, both verbal and non-verbal, and, in addition, given to dramatic modes of communication, to performance of different kinds. There are various types of social performance and genres of cultural performance, and each has its own style, goals, entelechy, rhetoric, developmental pattern, and characteristic roles. These types and genres differ in different cultures, and in terms of the scale and complexity of the socio-cultural fields in which they are generated and sustained." (Turner 1986: 82)

Besonders in diesem anthropologischen Ansatz sind enge Analogien zwischen der Theateraufführung als einer Form von cultural performance im herkömmlichen Sinne und der 'Aufführung' sozialer Situationen als social performance erkennbar. Für Turner sind die ritualisierten Formen der Interaktion in sozialen Alltagssituationen so dramenähnlich, dass er sie als social dramas bezeichnet. In der Ritualisierung dieser ,Alltagsdramen' lässt sich ihm zufolge eine Struktur erkennen, die der des literarischen stage drama gleicht, und zwar in ihrer ausgeprägten Form der des klassischen Dramas, wie Turner ausführt:

Typically, they have four main phases of public action. These are: (1) Breach of regular norm-governed social relations; (2) Crisis, during which there is a tendency for the breach to widen. [...] (3) Redressive action ranging from personal advice and informal mediation or arbitration to formal juridical and legal machinery, and [...] to the performance of public ritual [...] (4) The final phase consists either of the reintegration of the disturbed social group, or of the social recognition and legitimation of irreparable schism between the contesting parties. (ibid. 74f)

Im Sinne der eingangs beschriebenen Theatralität des Alltags lässt sich im Grunde für jede soziale Situation eine solche 'dramenästhetische' Struktur ermitteln, die allerdings auch, abweichend von Turners Annahme, wie 
verschiedene Formen des Dramas geschlossener oder offener sein kann. Denn ohne eine solche Annahme ließe sich eine soziale Interaktion als quasi-dramatische Situation weder (von den Teilnehmern selbst) konstituieren noch (von einem Beobachter) identifizieren.

Umgekehrt kann das social drama betrachtet werden als eine Art "experiential matrix from which the many genres of cultural performance, beginning with redressive ritual and juridical procedures, and eventually including oral and literary narrative, have been generated" (Turner 1974: 78). Diese Modellierung des Sozialen im Drama eröffnet dem Literaturunterricht eine wichtige reflexive Dimension, die es erlaubt, ein literarisches Drama als Kommentar zum eigenen sozialen Agieren zu lesen: "This proximity of theatre to life, while remaining at a mirror distance from it, makes of it the form best fitted to comment or 'meta-comment' on conflict, for life is conflict [... ]" (ibid. 105).

Erika Fischer-Lichte spricht wegen dieser Dramatizität oder Theatralität des Sozialen von einer Ärt der Wirklichkeitserfahrung", deren Modell das Theater darstellt: „Als Wirklichkeit (Theater) wird eine Situation erfahren, in der ein Akteur an einem - häufig besonders hergerichteten - Ort zu einer bestimmten Zeit vor den Blicken anderer (Zuchauer) etwas tut. Wirklichkeit erscheint in diesem Sinne prinzipiell als theatrale Wirklichkeit" (Fischer-Lichte 2002: 292). Diese sieht Fischer-Lichte von einer umfassenden Theatralisierung aller Sphären des öffentlichen, ja sogar des privaten Lebens geprägt; 'Wirklichkeit' wird im Wesentlichen als Inszenierung erfahren:

In allen gesellschaftlichen Bereichen wetteifern einzelne und gesellschaftliche Gruppen in der Kunst, sich selbst und ihre Lebenswelt wirkungsvoll in Szene zu setzen. Stadtplanung, Architektur und Design inszenieren unsere Umwelt als kulissenartige Ënvironments", in denen mit wechselnden Öutfits"kostümierte Individuen und Gruppen sich selbst und ihren eigenen Lifestylemit Effekt zur Schau stellen. [...] Eine schier endlose Abfolge von inszenierten Ereignissen weist darauf hin, daß sich eine Ërlebnis- und Spektakelkultur"gebildet hat, die sich mit der Inszenierung von Ereignissen selbst hervorbringt und reproduziert. In ihr wird Wirklichkeit mehr und mehr als Darstellung und als Inszenierung erlebt. (ibid. 291f.)

Ein von ihr besonders hervorgehobener Aspekt des Zusammenhangs von theatraler Inszenierung und Alltagshandeln steht in engem Bezug zur Lebenswelt der heranwachsenden Menschen im 21. Jahrhundert: Die neuen visuellen und elektronischen Medien verstärken den Inszenierungscharakter der Wirklichkeit erheblich; ganze Biographien, vor allem in der Populärkultur, sind nur noch als medial inszenierte konzeptualisierbar; "[m]ediale Simulationen, sogenannte virtuelle Realitäten, konkurrieren als mögliche Welten mit der empirischen Welt"(ibid. 293): 
Ob Musikstil, Mode, Markentreue, Tanzkulturen, Kommunikationsformen - wichtige Orientierungspunkte der jungen Generation werden nach kommerziellen Gesichtspunkten in kombinierten medialen Angeboten etabliert, verfestigt, aber immer auch wieder verändert. [...] Das Handeln und Verhalten der [medialen - W.H.] Akteure wird so wahrgenommen, daß es die eigene Aufführung des/der jungen Zuschauers/-in im Alltag zu modellieren vermag. (ibid. 24f.; vgl. auch Rosen 2007)

Das Verhalten in und der Umgang mit der solcherart theatralisierten, zunehmend medial durchdrungenen Wirklichkeit verstärken die Dringlichkeit der Entwicklung einer Performativen Kompetenz; nur sie kann die jungen Menschen befähigen, den Inszenierungscharakter verschiedener Wirklichkeitsebenen zu erkennen, sich selbst in diesen inszenierten Wirklichkeiten zu positionieren sowie selbstbestimmt und verantwortungsvoll damit umzugehen.

\section{Performative Kompetenz als Bildungsziel}

Wenn kulturelles Handeln Merkmale der Dramatizität und der Theatralität aufweist, so muss es auf Seiten der Akteure auch eine - Kompetenz genannte kognitive Disposition und kognitive Strukturen geben, die das Handeln in solchen Situationen steuern. Erst eine solche Performative Kompetenz ermöglicht den an sozialer Interaktion beteiligten Aktanten regelgeleitetes, strukturiertes soziales Handeln (also 'Performanz'): The phase structure of social dramas is not the product of instinct but of models and metaphors carried in the actors' heads. [... ] Structures are the more stable aspects of action and interrelationship" (Turner 1974: 36). Das Verstehen und die kognitive Aneignung dramatischer Strukturen sind also nicht primär literarische, sondern zuallererst soziale Fähigkeiten, die in situativ-sozialen Interaktionen zum Tragen kommen. Umgekehrt gilt auch: Das Verstehen literarischer Dramen (vornehmlich nicht durch Lesen, sondern durch Anschauung einer Aufführung) kann auf eine vorgängige 'dramatische' Kompetenz zurückgreifen, weil Dramen soziale Situationen modellieren.

Im schulischen Kontext ist die Entwicklung einer Performativen Kompetenz der Lernenden mit dem Ziel einer vollgültigen Teilnahme an sozialen und kulturellen Interaktionssituationen bisher allerdings weder als Bildungsziel noch als Bildungsinhalt bestimmt worden. Das hat seinen Grund u.a. vermutlich darin, dass die Vorstellung vom Inszenierungscharakter der kulturellen Wirklichkeit auf vergleichsweise junge kulturwissenschaftliche Ansätze rekurriert. ${ }^{2}$ Gleichwohl können all jene pädagogischen und didaktischen Ansätze, die in verschiedenen Fächern und schulischen Kontexten mit Konzepten von ,Inszenierung' und ,dramatischem Spiel' arbeiten, dem übergreifenden Konzept der

\footnotetext{
${ }^{2}$ Diese greifen interessanterweise kaum auf die frühen Ansätze z.B. Erving Goffmans (The Presentation of Self in Everyday Life, 1959) oder des in diesem Beitrag mehrfach zitierten Victor Turner zurück.
} 
Performativen Kompetenz zugeordnet werden, denn in ihnen allen geht es um die Inszenierung sozialer Interaktion und um die spielerische Erprobung individueller Performanzen. Performance, play und stage sind also Schlüsselkonzepte nicht nur im Bereich der dramatischen Literatur und des Theaters, sondern auch für die Entwicklung eines entsprechenden übergreifenden Bildungsziels jenseits der literarischen Bildung: Performative Kompetenz ist erforderlich, um als Aktant wirkungsvoll soziale Interaktionen initiieren und entwickeln zu können und die eigene Position im komplexen Zusammenspiel verschiedener Wirklichkeitsebenen zur Geltung bringen zu können. Das Fremdsprachenlernen gewinnt daher auch deutlich eine Dimension des kulturellen Lernens, das die Schüler/innen zur Bewältigung von Alltagssituationen in ihrer eigenen Lebenswelt befähigt (vgl. Hallet 2010b).

\section{Die performative Dimension des Fremdsprachenunterrichts}

Der Unterricht im Allgemeinen und der Fremdsprachenunterricht im Besonderen stellen selbst Wirklichkeiten mit eigenen Konstituierungsregeln dar, die verschiedene Merkmale der Inszenierung und des Performativen aufweisen. Die verschiedenen Ebenen dieser Performativität des Fremdsprachenunterrichts sollen im Folgenden kurz erläutert werden; sie werden dann in Teil 4 zusammenfassend in einem Einbettungsmodell zusammengeführt.

\subsection{Die Theatralität des Fremdsprachenunterrichts}

Wie jeder Unterricht stellt auch der Fremdsprachenunterricht eine besondere Form inszenierter Wirklichkeit dar mit spezifischen illokutiven Voraussetzungen", in dem ëin spezifischer thematisch oder situativ ausgerichteter Sprechakt in einen didaktischen eingebettet ist"(Hüllen 1976: 41). Das bestimmende Merkmal ist aufgrund der Setzung 'Fremdsprachigkeit' die Fiktionalität aller Äußerungen als "Beschränkung auf ein Sprachmedium, das nicht das natürliche Medium der sozialen Interaktion der daran beteiligten Menschen ist"(ibid. 43). Zu den Inszenierungsbedingungen und -regeln des Unterrichts gehört, dass „der Lehrer [... ] zugleich die Perspektive des Didaktischen und die Fiktion des thematischen Unterrichts aufrechterhalten [muß], der Lerner muß die ,Zumutung' des Fiktiven im Interesse des Didaktischen akzeptieren." (ibid. 44) Der Fremdsprachenunterricht stellt sich daher auch jenseits besonderer szenischer Verfahren als eine Bühne dar, auf der bereits auf der Ebene der 'Kommunikationsform Unterricht' ein diskursiv-soziales Spiel inszeniert wird. Noch genauer kann man im Sinne des Prinzips der Performativität sagen: Die spezifische Form der (fiktionalen) fremdsprachigen Kommunikation erzeugt den Fremdsprachenunterricht im Moment des sprachlich-diskursiven Vollzugs. Daher liegt es auf der Hand, dass 
Lehrende wie Lernende auch im Fremdsprachenunterricht selbst zur Aufrechterhaltung dieses Spiels über ein gewisses Maß an Performativer Kompetenz verfügen müssen, vor allem über die Fähigkeit, sich selbst vor und in einer Gruppe fremdsprachlich agierender Menschen zu artikulieren, sich in diesem halböffentlichen Raum zu positionieren und an den jeweiligen (meist fiktionalen) Aushandlungen in der fremden Sprache teilzunehmen.

\subsection{Szenisch-dialogische Texte im Fremdsprachenunterricht}

Zur Fiktionalität und Theatralität des Fremdsprachenunterrichts gehört eine lange Tradition des Umgangs mit szenisch-dialogischen Texten, die sich mannigfach im Repertoire aller Lehrwerke und in jedem Unterricht finden. Jenseits einer oft diskutierten Problematik, die in der Pseudo-Authentizität dieser Lehrbuch-Dialoge begründet liegt, weisen diese szenisch-dialogischen Lehrbuch-Texte eine unbestreitbare Ähnlichkeit mit literarischen Mini-Dramen und dramenähnliche Strukturen auf (vgl. das Beispiel oben sowie im Einzelnen Hallet 2008 und 2009). Auch der Umgang mit diesen kleinen Alltagsdramen erfordert Performative Kompetenz in einem dreifachen Sinn: zum ersten als Verstehen der in einem solchen Mini-Drama modellierten sozialen Interaktionssituation (Verstehens-Dimension), zum zweiten als Ausagieren dieses Mini-Dramas auf einer Bühne, und sei es auf der des Klassenzimmers (stage performance) oder als szenisches Lesen, und zum dritten als Konstituierung der hier vorgestellten Situation durch aktives eigenes Agieren in einer analogen Alltagssituation, also einem social drama (social performance).

\subsection{Die Inszenierungsdidaktik}

Dem Inszenierungscharakter des Fremdsprachenunterrichts ist in einem eigenständigen Ansatz Rechnung getragen worden, in dem das fremdsprachliche Klassenzimmer nicht nur metaphorisch, sondern real zu einer Bühne für fremdsprachige Darbietungen und Aufführungen wird. In einem solchen Konzept werden alle fremdsprachigen Äußerungen durch Rollenvergaben für fiktive Interviews, role-plays oder Simulationen zu einem expliziten, für die Lerner/innen transparenten und von ihnen mitentwickelten Teil des performativen Spiels des Fremdsprachenunterrichts. Ein europäisches Kooperationsprojekt unter dem Titel SStaging Foreign Language Learning. Inszenieren von Fremdsprachenlernen"hat diesen Ansatz zu einer umfassenderen Inszenierungsdidaktik entwickelt (Europäisches Kooperationsprojekt 2003, 2005). In Projekten für alle Klassenstufen der Sekundarstufe I sowie für die Themen Ïntercultural Competence", The Media", Performingünd "Words in Context"wurden Arbeitsbeispiele für die Lehrerfortbildung entwickelt, denen allesamt das Inszenierungs- und performance-Konzept gemeinsam ist. Die Autor/innen fassen es so zusammen:

Staging bedeutet für uns im Kern die Inszenierung erfolgreicher Lernprozesse bei Schülern und Lehrern in Form kreativer, prozess-, produkt- und 
kommunikationsorientierter Arbeits- und Lernszenarien in einer motivierenden, teilweise technologiebereicherten Lernumgebung. Ergebnisse werden dabei in kreativen mündlichen oder schriftlichen Präsentationen im Rahmen von Aufführungen oder Ausstellungen dargestellt. (Europäisches Kooperationsprojekt 2005, Projekte für die Klasse 5, 6 und 7: 7)

Hinsichtlich der Frage der Kompetenzentwicklung ist an diesem Ansatz besonders der letzte Aspekt relevant, der ausdrücklich mit Verweis auf die in der herkömmlichen 'Schulheft-Didaktik' fehlende Präsentationskompetenz begründet wird. Da sich viele Spielformen wie das job interview, Rollenspiele für alle möglichen realen Kontexte oder Video-Präsentationen und Internet-Auftritte an realen Situationen oder geläufigen medialen Formaten orientieren, lassen sich damit neben allgemeinen sprachlichen und kommunikativen Fähigkeiten besonders performative Fähigkeiten und Fertigkeiten entwickeln. In der Inszenierungsdidaktik ist Staging Lives daher ein Konzept zur Förderung lebensweltlicher und fremdsprachlicher Kompetenzen zugleich. Vor allem macht die inszenatorische Rahmung des Fremdsprachenunterrichts sichtbar, dass die Lernenden immer beiden Welten zugleich angehören, dem Fremdsprachenunterricht und der außerunterrichtlichen Welt. Jedes szenische Spiel, jedes Rollenspiel hat damit einen Doppelcharakter, indem es Kompetenzen für Lebenswelt und Unterricht gleichermaßen entwickelt (Abb. 1).

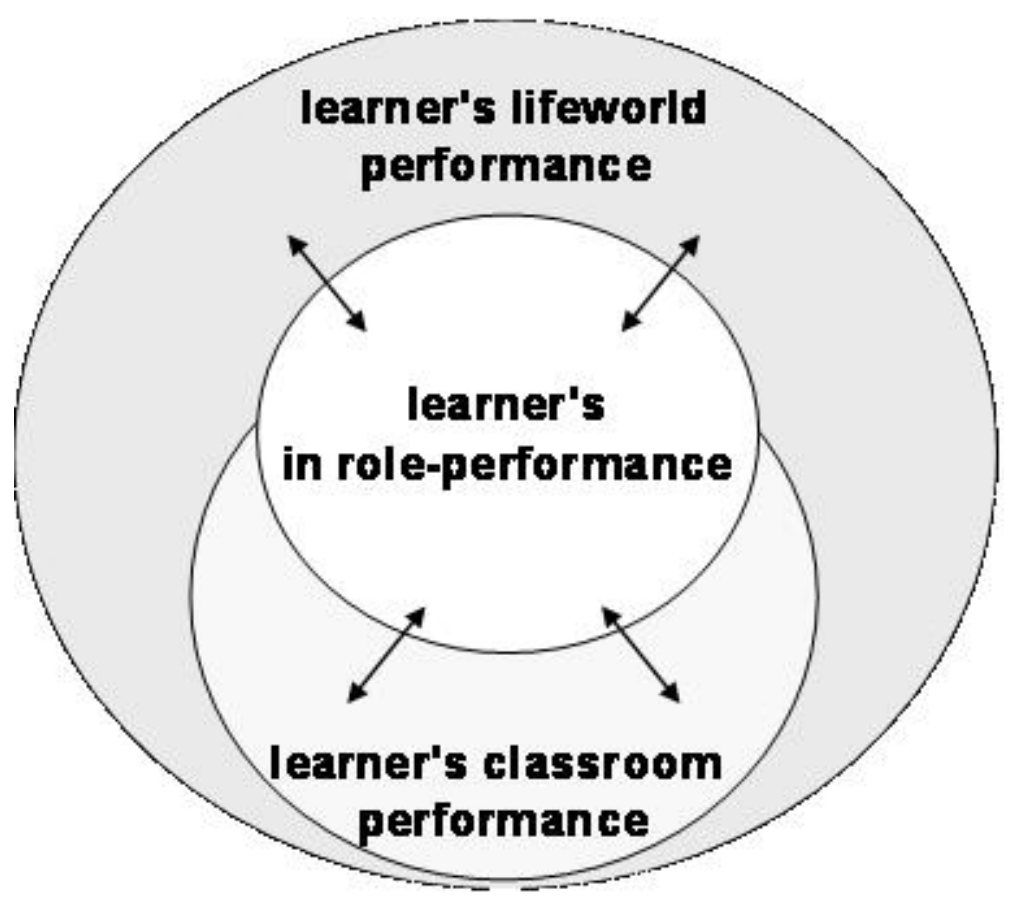

Abbildung 1: Die Förderung Performativer Kompetenz in Lebenswelt und Unterricht durch role-play. 


\section{4 'Inszenierung und Wirklichkeit' als Thema des Fremdsprachenunterrichts}

Vor allem die enorm gestiegene Bedeutung der visuellen und der elektronischen Medien hat dazu geführt, dass Formen der ästhetischen und der medialen Inszenierung durchgängig auch Themen des

Fremdsprachenunterrichts in Unterrichtseinheiten und Lehrwerken geworden sind. Eine solche thematische Beschäftigung mit den verschiedenen realen und medial inszenierten Wirklichkeiten ist für das Verstehen der von Werbung, Fernsehen, Internet und aller Arten von sogenanntem Live-Entertainment dominierten Lebenswelt der Heranwachsenden von zentraler Bedeutung. Erst diese Meta-Ebene erlaubt die kritisch-distanzierte und ethisch gegründete Sicht auf die Konstrukthaftigkeit und die Machart der verschiedenen Wirklichkeiten, deren Teil die jungen Menschen selbst sind. Von der Ausbildung einer entsprechenden fremdsprachlichen Begrifflichkeit einmal abgesehen, kann nur ein Blick hinter die Kulissen von Film und Fernsehen jene reflexive und ethische Dimension eröffnen, die ebenfalls Bestandteil der Performativen Kompetenz ist. Nur wer die Produktionsseite kennt, kann mediale performance wirklich verstehen, aber auch, wie in Video-Produktionen oder bei der Erstellung von Web-Seiten im Unterricht, mediale Auftritte selbst sinnvoll und verantwortungsvoll gestalten. Auch Schüler/innen der Sekundarstufe I können Reflexionen darüber selbst sehr früh leisten; sie kennen die medialen Welten oft besser als ihre Lehrer/innen und verfügen aufgrund eigener Erfahrungen durchaus über ein kritisches Denkvermögen, das mediale und soziale Erfahrungen zusammenzudenken weiß (vgl. dazu die Lernertexte in Hallet 2008).

\subsection{Dramatische Formen und szenische Verfahren}

Aus der oben beschriebenen Analogie von social und von stage drama gewinnt die Beschäftigung mit dramatischen Texten und Formen im Fremdsprachenunterricht eine neue Bestimmung: Um das Verstehen dramatischer Formen und Texte zu befördern, muss ihre soziale Welthaltigkeit einen größeren Stellenwert erhalten. Im Mittelpunkt der Beschäftigung müssen die Fragen stehen, die die sozialen und kulturellen Erfahrungen der Lernenden zu denen der Figuren in einem play in Beziehung setzt. Es handelt sich um solche Fragen, wie sie Turner für das social drama stellt: Welche Bhared values and interestsünd welche real or alleged common history"(Turner 1974: 69) verbindet die Figuren eines dramatischen Textes? Welcher Art ist der breach, der die Handlung und den nachfolgenden Konflikt auslöst? Wie und aus welchen Gründen wird daraus eine crisis? Welche Versuche von redress werden unternommen, von wem und aus welchem Interesse? Und schließlich: Was steht aus welchen Gründen am Ende, "either reintegration or recognition of schism"(ibid. 69)? Das Verstehen dramatischer Textes rekurriert also immer auf die sozialen Erfahrungen der Lernenden, und zugleich enthält der dramatische Text die Aufforderung, die 'Aufführung' eigener 
Lebenssituationen zu denen in einem Bühnendrama in Beziehung zu setzen.

Aus einem bloßen Text kann allerdings nur dann ein Drama werden, wenn die Lernenden aus den Buchstaben des Textes die Figuren, ihre Handlungen, ihre Denk- und Wertvorstellungen, ihre Verhaltensweisen und sogar deren körperliches Aussehen durch Aktivierung ihrer eigenen lebensweltlichen Erfahrungen kognitiv modellieren (vgl. genauer Hallet 2007) und in einem weiteren Schritt durch irgendeine Art der Aufführung zum Leben erwecken. Der Text eines Dramas ist also lediglich ein script für eine (mentale oder reale) Inszenierung durch die Lernenden; nur wenn die Texte auch ausagiert werden, werden die darin enthaltenen Wirklichkeits-, Handlungs- und Verhaltensmodelle auch sichtbar, und nur dann fungieren dramatische Texte auch "quasi als Metakommentare und Deutungsangebote zu sozialen Situationen und Konflikten, in die Menschen geraten können.SSo verstanden, können sie den Lernenden helfen, ßich selbst und das eigene Verhalten in sozialen Situationen zu reflektieren und gegebenenfalls zu verändern"(Scheller 2004: 23f.). Dramatische Texte 'zeigen' oder 'spiegeln' soziale Situationen also nicht nur, sondern sie stellen zugleich auch die generischen Muster, die available designs für die performative Erzeugung sozialer Interaktion in realweltlichen Kontexten bereit und sind daher in der sozialen Interaktion wahrnehmungssteuernd und strukturbildend (vgl. im Einzelnen Hallet 2009).

\section{Inszenierte Wirklichkeiten und Performative Kompetenz}

Es sollte deutlich geworden sein, dass Performative Kompetenz im weitesten Sinne die Fähigkeit des Verstehens von und der Partizipation an inszenierter Interaktion bedeutet. Aktive Partizipation an sozialer Interaktion setzt die Fähigkeit voraus, Situationen bzw. ihre Tiefenstruktur (im Sinne Turners) zu 'lesen', um sie (mit)konstituieren und in ihnen verantwortungsvoll, d.h. vor allem unter Respektierung der Positionen der anderen Teilnehmer/innen, agieren zu können. Es versteht sich, dass dazu auch die Fähigkeit gehört, aktiv an fremdsprachigen Diskursen der Konstitution von Wirklichkeit (also an fremdsprachigem staging lives) teilzuhaben. Von daher kommt der Tatsache eine besondere Bedeutung zu, dass der Fremdsprachenunterricht nicht nur selbst inszenierte Wirklichkeit ist, sondern dass er zugleich eingebettet ist in und bezogen auf die soziale und mediale Inszenierung von lebensweltlichen Wirklichkeiten, die ihrerseits fremdsprachig geprägt und damit mehrsprachig sind. Dies ist die eigentliche Begründung dafür, dass die theatrale und performative Dimension von Wirklichkeit innerhalb des Fremdsprachenunterrichts auf verschiedenen Ebenen zur Geltung kommen muss (vgl. Abb. 2), und zwar

- thematisch als Gegenstand des Unterrichts,

- in Gestalt didaktischer szenisch-dialogischer Texte, 
- in Gestalt literarischer dramatischer Texte,

- durch die Verwendung szenisch-performativer Formen (Rollenspiel etc.) und

- schließlich in komplexer Form durch die Inszenierung von stage dramas.

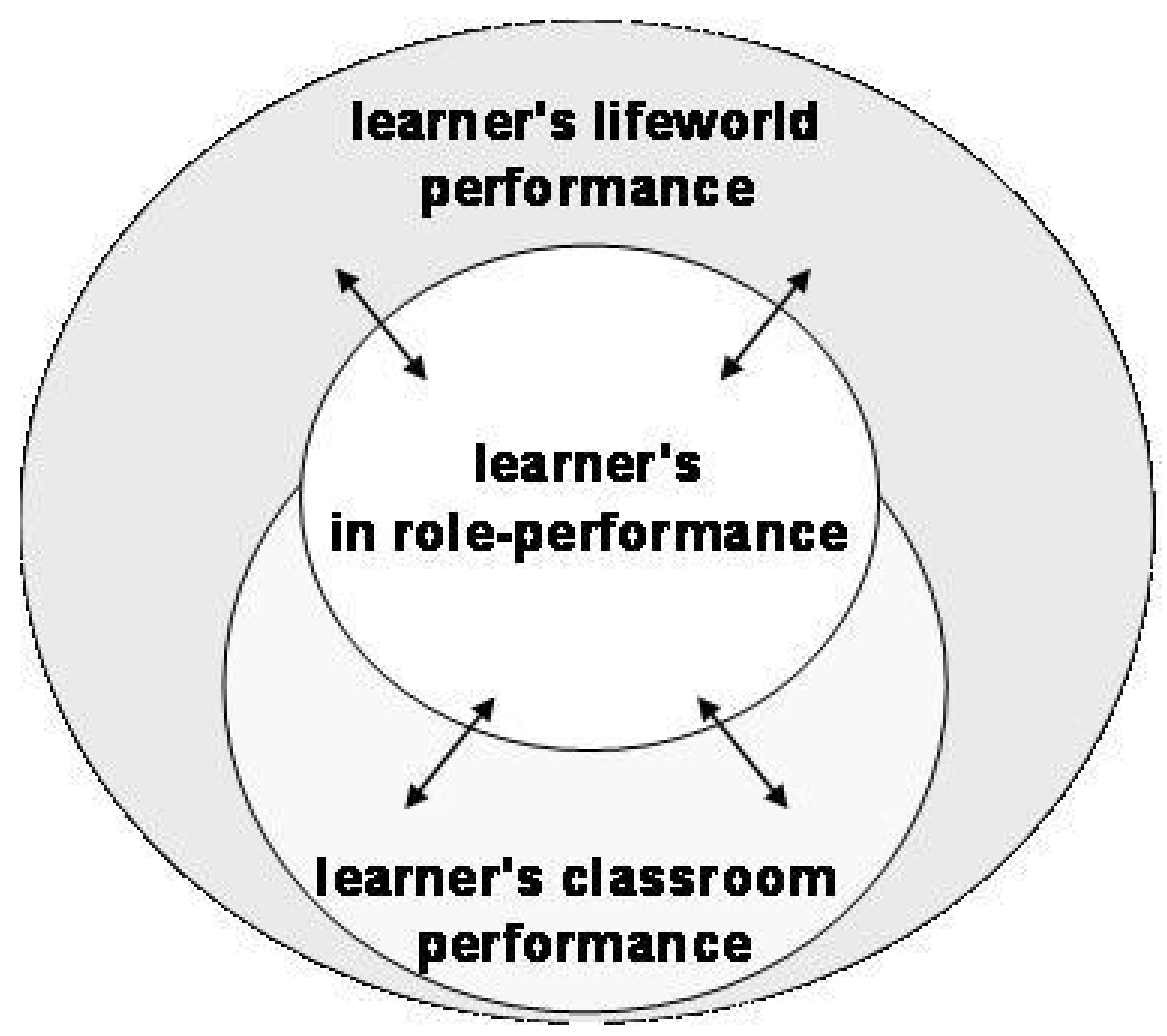

Abbildung 2: Inszenierte Wirklichkeiten und Performative Kompetenz.

Alle diese Ebenen tragen in unterschiedlicher Weise zur Entwicklung Performativer Kompetenz bei. Sie lässt sich zusammenfassend als Bündel folgender Fähigkeiten definieren: als Fähigkeit zur fremdsprachigen

- aktiven, verantwortungsvollen, partnerschaftlichen Partizipation an sozialen Interaktionen und Aushandlungen;

- kognitiven und handelnden Strukturierung von social dramas, role-taking usw.;

- Identifizierung und Bestimmung von Interaktionsrollen (auch der eigenen);

- situationsadäquaten performance in verschiedenen Kontexten, auch im Unterricht; 
- kritischen (auch ethischen) Reflexion eigener Inszenierungen und 'Auftritte';

- Unterscheidung verschiedener Wirklichkeitsebenen und die kritische (auch ethische) Reflexion von Inszenierungen, besonders auch von medialen

- sowie als Fähigkeit

- zum Verstehen der Interaktionsmodelle und der Rhetorik von stage dramas und dramatischen Texten.

Die Entwicklung und die Förderung der Performativen Kompetenz im Fremdsprachenunterricht erfordern vor allem einen deutlichen Akzent auf der Mündlichkeit und auf der 'Inszenierung' von Interaktionssituationen, in denen die Lernenden probeweise, d.h. auch in einem geschützten, nicht den kommunikativen Ernstfall darstellenden Raum, und in der Fremdsprache (mit der ihr innewohnenden Fiktionalität und Distanzierung vom sozialen Ernstfall) ihre 'Auftritte' planvoll inszenieren, ausagieren und beobachtend reflektieren können. Von kleinen Lehrwerkdialogen nach dem Muster des oben zitierten Beispiels über Rollenspiele und komplette Minidramen bis hin zu komplexeren dramatischen Texten sind hier alle Formen der fremdsprachigen Interaktion denkbar und sinnvoll. Im Sinne der Analogie von stage drama und social drama ist es wichtig, dass die Lernenden eine Vorstellung von der dramatischen Struktur sozialer Interaktionen, also von deren generischem Charakter gewinnen: Die soziale Interaktion lässt sich ebenso als Genre begreifen wie im Schriftlichen etwa der Zeitungsbericht oder die Erörterung. Der Erwerb der Performativen Kompetenz muss daher auch als generisches Lernen, als Aneignung der interaktionalen und diskursiven Verlaufsformen von sprachlicher und sozialer Interaktion gestaltet werden (vgl. Hallet 2009). Da auch literarische dramatische Texte soziale 'In-Szene-Setzungen' modellieren, tragen auch das literarische Verstehen und das szenische Ausagieren von literarischen Figuren entscheidend zur Entwicklung der Performativen Kompetenz bei (vgl. genauer Hallet 2007). In einem so verstandenen Literaturunterricht sind die Ästhetik des literarischen Textes und die Rhetorik der sozialen Situation nicht zu trennen. Daher ist auch die ästhetische Bildung zugleich soziale und kulturelle Bildung, und umgekehrt.

Literatur -

Butler, Judith (2002): Performative Akte und Geschlechterkonstitution. Phänomenologie und feministische Theorie. In: Wirth, Uwe (Hrsg.): Performanz. Zwischen Sprachphilosophie und Kulturwissenschaften. Frankfurt/Main: Suhrkamp, 301-320. 
Europäisches Kooperationsprojekt (2003, 2005): Staging Foreign Language Learning. Inszenieren von Fremdsprachenlernen. Inszenierung als Methode im Fremdsprachenunterricht. Bände: Intercultural Competence; The Media; Performing; Words in Context, alle Berlin: Cornelsen (2003). Projekte für die Klassen 5, 6 und 7; Projekte für die Klassen 8, 9 und 10, beide Berlin: Cornelsen (2005).

Fischer-Lichte, Erika (2004): Einleitung. Theatralität als kulturelles Modell. In: Fischer-Lichte, Erika; Horn, Christian; Umathum, Sandra; Warstat, Matthias (Hrsg.): Theatralität als Modell in den Kulturwissenschaften. Tübingen/Basel: Francke, 7-26.

Fischer-Lichte, Erika (2002): Grenzgänge und Tauschhandel. Auf dem Wege zu einer performativen Kultur. In: Wirth, Uwe (Hrsg.): Performanz. Zwischen Sprachphilosophie und Kulturwissenschaften. Frankfurt/Main: Suhrkamp, 277-300.

Goffman, Erving (1959): The Presentation of Self in Everyday Life. Garden City, NY: Doubleday Anchor.

Green Line 3 (2007). Hrsg. v. Harald Weisshaar. Stuttgart \&Leipzig: Klett.

Hallet, Wolfgang (2010a): Performative Kompetenz. In: Surkamp, Carola (Hrsg.): Lexikon Fremdsprachendidaktik. Stuttgart/Weimar: Metzler. [im Druck].

Hallet, Wolfgang (2010b): Kulturelles Lernen. In: Hallet, Wolfgang; Königs Frank G. (Hrsg.). Handbuch Fremdsprachendidaktik. Seelze-Velber: Klett-Kallmeyer, 129-133.

Hallet, Wolfgang (2009): Available Design. Kulturelles Handeln, Diskursfähigkeit und generisches Lernen im Englischunterricht. In: AbendrothTimmer, Dagmar; Lütge, Christiane; Elsner, Daniela; Viebrock, Britta (Hrsg.): Handlungsorientierung im Fokus: Impulse und Perspektiven für den Fremdsprachenunterricht im 21. Jahrhundert. Frankfurt/Main: Lang, 117-142.

Hallet, Wolfgang (2008): Staging Lives. Die Entwicklung performativer Kompetenz im Englischunterricht. In: Ahrens, Rüdiger; Eisenmann, Maria; Merkl, Matthias (Hrsg.): Moderne Dramendidaktik für den Englischunterricht. Heidelberg: Winter, 387-408.

Hallet, Wolfgang (2007): Literatur, Kognition und Kompetenz. Die Literarizität kulturellen Handelns. In: Bredella, Lothar; Hallet, Wolfgang (Hrsg.): Literaturunterricht, Kompetenzen und Bildung. Trier: Wissenschaftlicher Verlag Trier, 31-64. 
Hallet, Wolfgang; Hebel, Udo (2007): Staging Women's Lives: Short Plays im Englischunterricht. In: Der fremdsprachliche Unterricht Englisch 4, 85/86, 4-11.

Hüllen, Werner (1976): Linguistik und Englischunterricht 2. Didaktische Analysen. Heidelberg: Quelle \& Meyer.

Rosen, Larry D. (2007): Me, MySpace and I. Parenting the Net Generation. New York \& Houndmills: Palgrave Macmillan.

Scheller, Ingo (2004): Szenische Interpretation. Theorie und Praxis eines handlungs- und erfahrungsbezogenen Literaturunterrichts in Sekundarstufe I und II. Seelze: Kallmeyer.

Turner, Victor (1986): The Anthropology of Performance. New York: Performing Arts Journal.

Turner, Victor (1974): Dramas, Fields, and Metaphors. Symbolic Action in Human Society. Ithaca and London: Cornell UP. 